\title{
Barriers to the Integration of Psychosocial Factors in Medicine: Results of a National Survey of Physicians
}

\author{
John A. Astin, PhD, Karen Soeken, PhD, Victor S. Sierpina, MD, and \\ Brian R. Clarridge, $P h D$
}

Purpose: Examine physicians' attitudes toward the incorporation of psychosocial factors in diagnosis and treatment and identify barriers to the integration of evidence-based mind-body methods.

Method: Random sample of primary care physicians and physicians from selected non-primary specialties was drawn. A total of 1058 physicians completed a 12-page survey.

Results: The response rate was $\mathbf{2 7 \%}$. Although a majority of physicians seem to recognize the importance of addressing psychosocial issues, approximately one third believe that addressing such factors would lead to minimal or no improvements in outcomes. A minority reports their training regarding the role of psychosocial factors was effective, and relatively few indicate interest in receiving further training in these areas.

Males were less likely to believe in the importance of addressing psychosocial factors. Additional factors included perceptions that training was poor in these areas; feelings of low self-efficacy to address psychosocial issues and the perception that such factors are difficult to control; lack of knowledge of the evidence-base supporting the role of psychosocial factors; and lack of time and inadequate reimbursement to address the psychosocial domain.

Conclusions: These results suggest the need for more comprehensive training in the role of psychosocial factors in health. In addition, the finding that physicians identify lack of time and inadequate reimbursement as significant barriers suggests that the current health care delivery system may, in many respects, be antithetical to the biopsychosocial model. (J Am Board Fam Med 2006;19:557-65.)

The call for greater recognition of the role of psychological and sociocultural factors in medicine is certainly not new. Engel $^{1}$ published a seminal article almost 30 years ago calling for an expansion of the biomedical model to a biopsychosocial one. However, despite such calls for reform, studies suggest that psychosocial factors continue to be overlooked or frequently missed in clinical encounters $^{2-7}$ and tend to still be underemphasized in

This article was externally peer reviewed.

Submitted 12 March 2006; revised 6 July 2006; accepted 18 July 2006.

From the California Pacific Medical Center, San Francisco, CA (JAA), University of Maryland School of Nursing, Baltimore, MD (KS), University of Texas Medical Branch (VSS), and Center for Survey Research, University of Massachusetts, Boston, MA (BRC).

Funding: This work was supported by National Institutes of Health National Center for Complementary and Alternative Medicine Grant R01AT00869 to 04 (JAA), and National Institutes of Health Mind Body Exploratory and Development Grant 1R21AG023951-01 (VSS).

Conflict of interest: none declared.

Corresponding author: John A. Astin, $\mathrm{PhD}$, California $\mathrm{Pa}-$ cific Medical Center, 2300 California Street, Room 207, San Francisco, CA 94115 (E-mail: john@integrativearts.com). medical education. ${ }^{8,9}$ Studies also suggest that empirically supported mind-body interventions for such common health problems as pain and insomnia ${ }^{10,11}$ are used by only a minority of patients suffering from these conditions. ${ }^{12}$

Consistent with these findings, in October 2004, the National Institutes of Health issued a request for applications (RFA) focused on "strengthening behavioral and social science curricula in medical school." This RFA grew out of an Institute of Medicine report issued earlier that same year in which the authors noted that "no physician's education would be complete without an understanding of the role played by behavioral and social factors in human health and disease, knowledge of the ways in which these factors can be modified, and an appreciation of how personal life experiences influence physician-patient relationships."

Although there is a growing body of evidence pointing to the role that psychosocial factors such as stress can play in health and illness, and the well-accepted, contemporary value that medical practice should be grounded in solid scientific evi- 
dence, it is also well documented that evidence of a given therapy's effectiveness is frequently insufficient to change clinical practice. ${ }^{13}$ Therefore, if medical training and practice are to move toward a model that gives adequate attention to nonbiological (ie, psychosocial) concerns, the varied and complex barriers to integration must be identified and addressed. ${ }^{13,14}$

The present study reports the results of a national survey of physicians that was designed to examine attitudes toward the role of psychosocial factors (eg, stress, emotional states) in medicine, and to identify factors that might account for differences in the extent to which physicians recognize the importance of such factors in health and are open to using mind-body approaches (such as relaxation, stress management, meditation, and behavioral counseling) in clinical practice. ${ }^{11,12}$ More specifically, the study aimed to identify personal, attitudinal, and social-environmental factors that facilitate or inhibit physicians' openness to considering psychosocial factors in diagnosis and treatment, and to seek a better understanding of the role that medical education plays in shaping physicians' attitudes and practice patterns relative to these areas.

\section{Method}

In collaboration with researchers from the Center for Survey Research (CSR) at the University of Massachusetts, Boston, we obtained a list of physician mailing addresses from the American Medical Association (AMA) master files. This list of potential respondents was drawn from a broad spectrum of primary care specialties and a select group of non-primary care specialties. Primary care specialties included family medicine, internal medicine, pediatrics, and obstetrics/gynecology. Several nonprimary care specialties were selected including rheumatology, gastroenterology, pain management, and cardiology. The principle we applied was to include specialties that were in the front lines of primary care and/or whose practitioners might reasonably confront symptoms and diagnose conditions for which evidence-based mind-body methods could be considered appropriate adjuncts to care. ${ }^{11}$

In May 2002, we conducted a series of focus groups with physicians $(\mathrm{N}=22)$. This information was used, in part, to inform both domain and item selection for the survey. ${ }^{15}$ Once the questionnaire was formatted, CSR conducted cognitive interviews (via telephone) with 20 physicians who completed the measure to assess whether any items needed to be revised or omitted. This feedback resulted in several minor revisions that simplified the questionnaire's administration and clarified certain test items. The final questionnaire was 12 pages in length and took approximately 20 minutes to complete. It contained 36 items that along with assessing basic demographic information, asked respondents about their attitudes toward the role of psychosocial factors in health, their perceptions of training in these areas, their use of mind-body methods in clinical practice, and their perspective on factors that might serve as barriers to the integration of such methods. A Web-based version of the survey was also developed, giving respondents the option (in the mailed cover letter) to take the survey on-line at a designated URL.

There are a number of terms that are used (often interchangeably and at times with slightly different connotations) by both researchers and clinicians to describe the general topic area we were interested in exploring. Examples of these include: "mindbody medicine," "behavioral medicine," "psychosocial factors," and "biopsychosocial medicine." So as to minimize any confusion that might have resulted from our use of terms that respondents were either not familiar with, or for which they held different interpretations, the questionnaire began as follows:

This survey asks for your views on the psychosocial aspects of patient care. The information you provide will help us clarify the current status of mind-body medicine among practicing physicians. The terms "psychosocial" and "mind-body" refer to those approaches that emphasize the role of nonphysical factors such as stress, emotions, attitudes, and beliefs in the diagnosis and treatment of physical illness.

Initially, a random sample of 3350 physicians (from the above disciplines) was drawn from the AMA master files. Of these, 3057 were deemed interviewable (eg, appropriate specialty, valid telephone number or address). Physicians were mailed the survey, along with a cover letter, fact sheet, and return envelope. A small monetary incentive was to be included in the initial mailing but because of a clerical error at CSR, these were omitted. To test 
what the effect of including an incentive would have been, CSR contributed an additional random sample of 1000 physicians and mailed surveys with $\$ 20$ incentives to these potential respondents. For both groups (incentive and no incentive), reminder telephone calls were begun approximately 14 days after the initial mailing. A second questionnaire packet was sent to all nonresponders, followed by another series of follow-up phone calls.

\section{Results}

\section{Response Rate}

Of the original 3057 mailed surveys, 683 completed responses were received (22\%). A total of 18 physicians $(2.7 \%)$ opted to use the Web-based version of the survey. For the second, incentivized mailing, completed surveys were received from 375 of 873 eligible physicians constituting a significantly higher response rate of $43 \%$. The combined sample used for all descriptive and multivariate analyses is $\mathrm{N}=1058$, (overall response, $27 \%$ ).

Tests for nonresponse bias indicate that among physicians receiving no incentive, females were more likely to respond, as were those who completed medical school after 1995. For this reason, we weighted the nonincentivized sample to adjust for these potential biases. Further analyses of the 2 samples (incentivized and nonincentivized) indicated that respondents who did not receive a monetary incentive tended to hold slightly more favorable attitudes toward the topic area (ie, the importance of incorporating psychosocial factors in training and practice). For this reason, we added "receipt of incentive" as a covariate in all analyses to control for this potential bias.

\section{Descriptive Statistics}

Demographics

Table 1 summarizes the demographic characteristics of the combined sample. Mean age is 48.9 . Sixty-nine percent are male; $31 \%$ are female. The largest specialty groups represented are: family medicine $(20 \%)$, internal medicine $(19 \%)$, pediatrics (18\%), and obstetrics/gynecology (14\%). Additional non-primary care specialties include cardiology, dermatology, physical medicine rehabilitation, rheumatology, and pain medicine.

\section{Use of Mind-Body Methods}

The majority of physicians seem to recognize the importance of addressing the psychosocial domain
Table 1. Sample Characteristics

\begin{tabular}{lc}
\hline Demographic Data & Percentage \\
\hline Year graduated from medical school & \\
Before 1960 & 4.9 \\
1960-1969 & 11.4 \\
1970-1979 & 27.0 \\
1980-1989 & 36.2 \\
1990-2001 & 20.4 \\
Sex & \\
Female & 31.3 \\
Male & 68.7 \\
Religious affiliation & \\
Protestant & 36.1 \\
Catholic & 25.5 \\
Jewish & 14.4 \\
Hindu & 3.5 \\
Muslim & 2.7 \\
Buddhist & 1.2 \\
No religious affiliation & 11.0 \\
Medical specialty & \\
Family practice & 20.3 \\
Internal medicine & 18.6 \\
Pediatrics & 18.4 \\
Obstetrics/gynecology & 13.5 \\
Cardiology & 4.9 \\
Dermatology & 3.7 \\
Physical medicine/rehabilitation & 3.1 \\
Other* & \\
\hline
\end{tabular}

* Urology, gastroenterology, rheumatology, allergy, and pain medicine.

in clinical practice. For example, in response to the question, "What would be the overall improvement in treatment outcomes from increasing the application of psychosocial methods," two thirds (66\%) indicate that including such methods would lead to "moderate" or "big" improvements (score of 3 or 4 on a 4-point scale), compared with $34 \%$ who state that such an addition would lead only to "small" or "almost no" improvements. Analysis of the 4 primary care specialties included in our survey showed small but nonsignificant differences across groups, with $70 \%$ of family medicine physicians indicating that psychosocial methods would significantly improve treatment outcomes, followed by pediatrics (68\%), internal medicine (67\%), and obstetricsgynecology (65\%).

Respondents were given a list of representative mind-body interventions and asked to comment on the extent to which they used and/or referred out to each of these as part of their clinical practice. As 


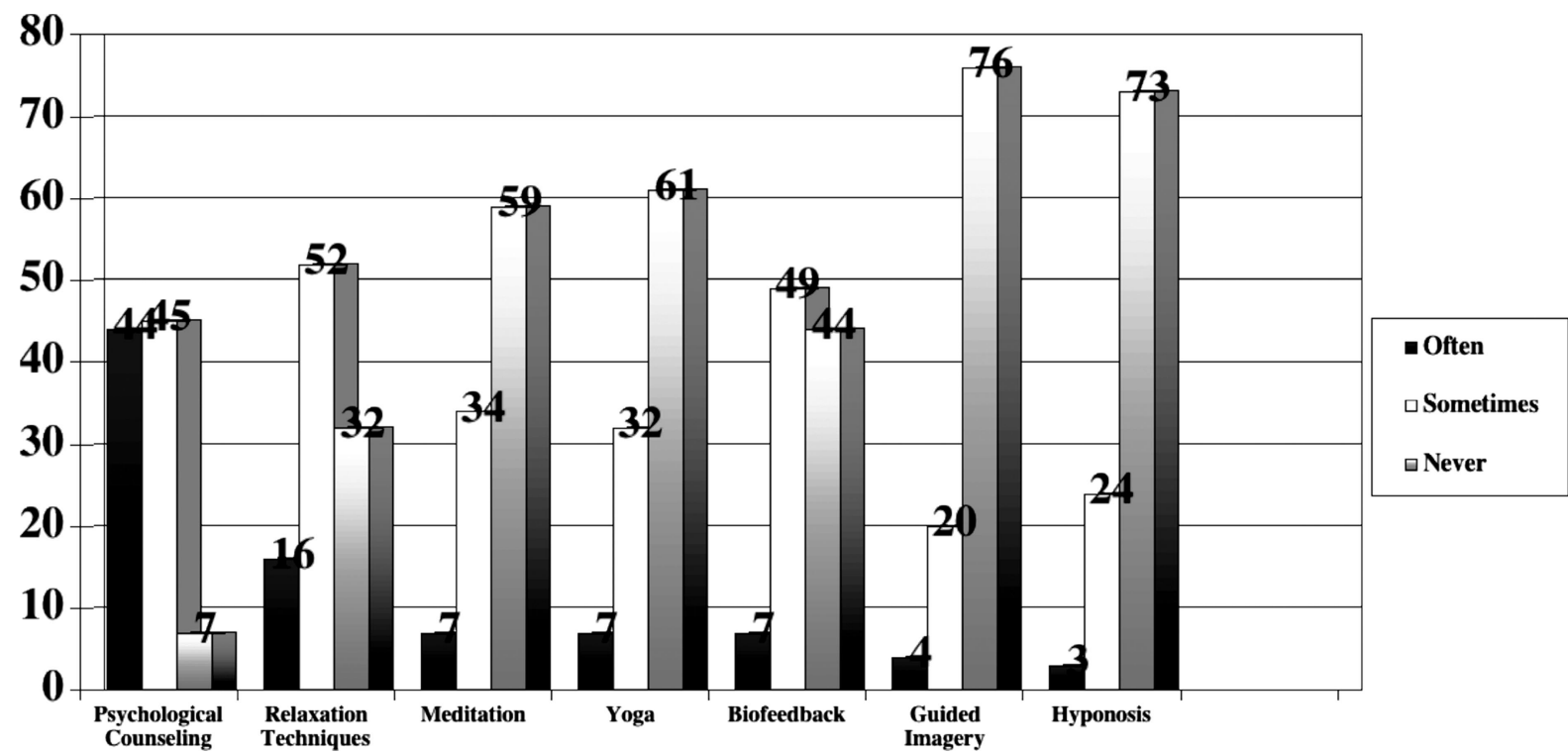

Figure 1. Use of/Referral for Mind-Body Methods.

shown in Figure 1, psychological counseling and relaxation techniques are the most commonly used therapies with the majority of physicians reporting use of and/or referral to counseling (44\% often; $45 \%$ sometimes) and relaxation therapies (16\% often; $52 \%$ sometimes).

\section{Perceptions about Training}

A minority (25\%) of respondents indicate that their formal training (medical school, residency) was "helpful" (score of 4 or 5 on a 5 -point scale) in learning how to address the psychosocial domain (either diagnostically or in terms of actual treatment), whereas $44 \%$ rate the quality of their training in these areas as "not helpful" (score of 1 or 2) (see Figure 2).
Forty-three percent indicate that mentors in medical school did a good job with respect to diagnosing psychosocial factors, whereas $24 \%$ say that teachers effectively mentored them about including mind-body methods in treatment. Approximately half of the respondents (49\%) indicate that they received effective mentoring during residency in diagnosing psychosocial issues, whereas only 1 in $3(33 \%)$ indicate that residency mentors did a good job training them in the clinical application of mind-body methods.

\section{Behavioral Intentions}

To assess physicians' future intentions with respect to incorporating mind-body methods, we asked them to comment on their level of interest in re-

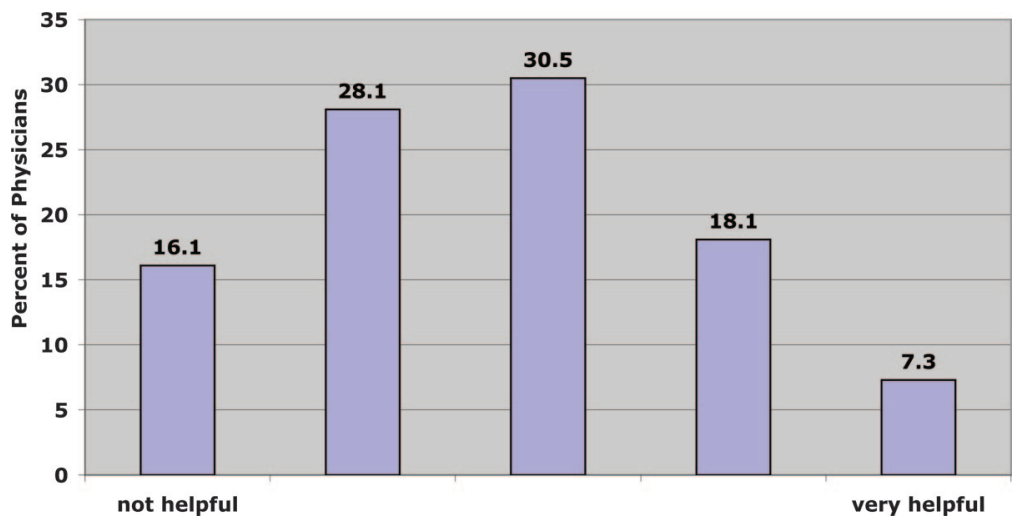

Figure 2. Usefulness of Formal Medical Training to Include Psychosocial/Behavioral Methods in Treatment. 


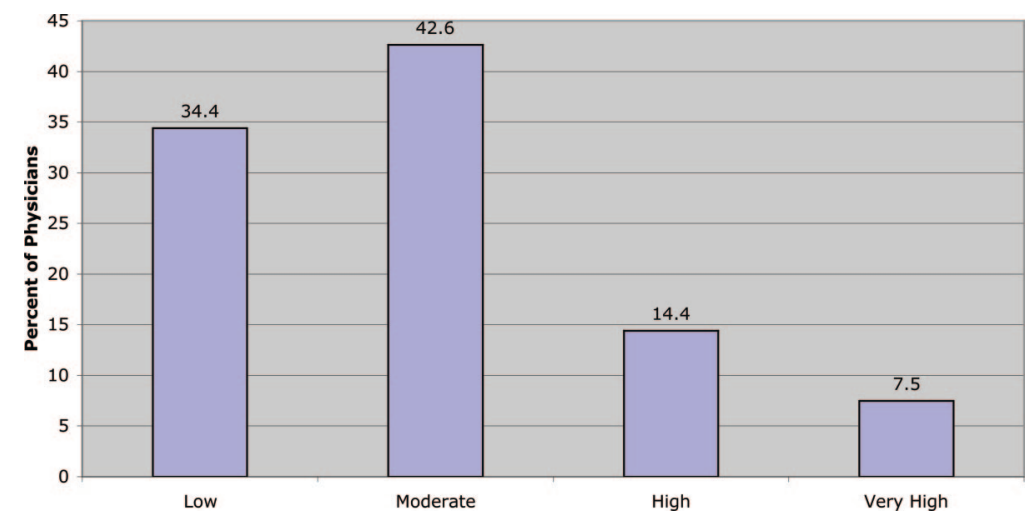

Figure 3. Level of Interest in Receiving Further Training in Mind-Body Medicine.

ceiving further training in these areas, and the extent to which they felt committed to incorporating mind-body approaches clinically. As shown in Figure 3, a minority, $22 \%$, express high or very high interest (3 or 4 on a 4 -point scale) in obtaining additional training, with the remaining report either moderate (44\%) or low (34\%) levels of interest in receiving further mind-body training. Approximately one third of respondents indicate that they are either "not very committed" $(25 \%)$ or "not at all committed" (8\%) to such adoption, whereas $17 \%$ report being "very committed" and 49\% "somewhat committed."

\section{Multivariate Analyses}

In an effort to better understand potential barriers to the integration of the biopsychosocial model in medicine, we examined the extent to which selected variables predict both attitudes toward, and practice of, mind-body methods. Based on our review of the literature and the results from the focus groups we had previously conducted, ${ }^{15}$ we hypothesized that the following predictors would be significant in the multivariate analyses: gender; year graduated medical school; medical specialty; extent of belief in the evidence-base for mind-body methods; lack of clinical expertise (ie, low self-efficacy); perceptions of control (ie, inability to influence psychosocial factors); lack of time to address psychosocial issues; degree of social (peer) support; importance of religion/spirituality, and personal use of mind-body approaches.

Because of missing data on some questionnaire items, the overall $\mathrm{N}$ (listwise present) for the regression analysis was 948, which represented 90\% of the completed surveys. Of those excluded from this analytic sample, most (75\%) failed to respond to only one of the variables included in the multivariate analyses. Two dependent variables were considered: belief in the "value-added" of mindbody methods ("Attitude"), and current clinical use of/referral to mind-body therapies ("Practice"). These variables are significantly correlated: $r=$ $0.43(P<.001)$. In all regression analyses, diagnostics indicate no evidence of multicollinearity and the plot of standardized residuals suggested a normal distribution with fewer than $1 \%$ of standardized residuals exceeding an absolute value of 3.0. Three cases, only $0.3 \%$ of the sample, were identified as multivariate outliers based on Mahalanobis distance but given the small number, they were not deleted for analyses.

\section{Predictors of Attitude}

When the dependent variable, belief in the valueadded of mind-body methods ("Attitude"), was examined, the following variables emerged as statistically significant in the regression (see Table 2): 1) belief that "the absence of demonstrably effective mind-body techniques" limits use $(\beta=.28), 2)$ "usefulness of formal medical training" $(\beta=.18)$, 3) personal use of mind-body therapies $(\beta=.15), 4)$ being female $(\beta=.15), 5)$ belief that lack of expertise does not limit use $(\beta=-.07)$, and 6$) \mathrm{im}$ portance of religious/spiritual beliefs $(\beta=.06)$. Together, these factors explain $21.4 \%$ of the variability in attitude toward mind-body issues (adjusted $\left.R^{2}=0.205\right)$. These results indicate that physicians who perceive increased value-added when mind-body methods are used in concert with other medical therapies are more likely to be female, rate their formal education as more useful in addressing 
Table 2. Predicting Physicians' Belief that Incorporating Mind-Body Approaches Would Improve the Prevention and Treatment of Common Medical Conditions*

\begin{tabular}{lcc}
\hline Significant Predictors & $\beta$ Coefficient & $P$ Value \\
\hline $\begin{array}{l}\text { Belief that a lack of evidence } \\
\text { represents a significant } \\
\text { barrier }\end{array}$ & -.275 & $<.001$ \\
$\begin{array}{l}\text { Belief that formal training } \\
\text { regarding the role of } \\
\text { psychosocial factors was }\end{array}$ & .179 & $<.001$ \\
$\quad$ useful & & \\
$\begin{array}{l}\text { Use mind-body methods to } \\
\text { manage own health }\end{array}$ & .153 & $<.001$ \\
$\begin{array}{l}\text { Gender (female) } \\
\text { Belief that lack of expertise is a } \\
\text { barrier }\end{array}$ & -.07 & $<.001$ \\
$\begin{array}{l}\text { Report that spiritual beliefs are } \\
\text { important to them in their }\end{array}$ & .064 & $<.05$ \\
$\quad$ work & & \\
\end{tabular}

* Physicians' rating of the usefulness of mind-body methods for insomnia, low back pain, arthritis, cardiovascular disease, hypertension, headache, combined into a single score.

psychosocial issues, use mind-body methods for their own health more often, state that their spiritual/religious beliefs are important to them in their work, not feel that the absence of evidence (of efficacy) limits their use of mind-body approaches, and believe that "lack of expertise" does limit the use of such methods.

\section{Predictors of Practice}

When use of and/or referral to mind-body therapies ("Practice") was tested as the dependent variable in the regression, the following factors emerged as significant predictors: personal use of mind-body techniques $(\beta=.17)$, "usefulness of formal medical training" $(\beta=.11)$, "lack of expertise" $(\beta=-.17)$, "absence of demonstrably effective mind-body techniques" ( $\beta=-.09)$, "insufficient clinic time" $(\beta=-.08)$, and "belief that psychosocial factors are beyond one's capacity to control or influence" $(\beta=-.07)$. The model fit was again significant $(P<.001)$ with $20.3 \%$ of the variability explained by this set of predictors. These results indicate that physicians who use and/or refer to mind-body therapies more frequently in clinical practice tend to report using such methods for their own health, rate their formal training as more helpful, and are less likely to say that lack of expertise, poor evidence of efficacy, insufficient clinic time, and low feelings of perceived control (to influence psychosocial factors) are significant obstacles. Neither medical specialty nor year graduated medical school predicted either attitude toward or practice of psychosocial/mind-body methods.

As noted above, being female, using mind-body methods for one's own health, and believing that training in medical school and residency effectively addressed these areas, each emerged as significant predictors of greater openness to and/or actual clinical use of mind-body methods. For example, $77 \%$ percent of female physicians, compared with $61 \%$ of their male counterparts, believe that the addition of mind-body methods would lead to significant improvements in treatment outcomes. Among those who report using such methods to address their own health issues, $78 \%$ say that integrating mind-body methods would lead to significant improvements in treatment outcomes compared with $51 \%$ of those who do not personally use such methods. We note that female physicians are also more likely to use such methods to manage their own health $(66 \%$, compared with $50 \%$ of men).

Among physicians who rate their formal training as "not helpful," $55 \%$ state that the inclusion of mind-body methods would lead to improved treatment outcomes, compared with $84 \%$ of physicians who consider their training in these areas to have been "very helpful." Interestingly, physicians who rate their training in the role of psychosocial factors more positively are also more likely $(30 \%$, compared with $16 \%$ ) to express a strong interest in receiving further training in mind-body methods.

\section{Discussion}

The present study sought to examine physician' attitudes toward the role of psychosocial factors and mind-body methods in medicine. An additional goal was to identify factors that might account for physicians' relative openness and willingness to integrate a more biopsychosocial perspective into the way they view and ultimately practice medicine. Several themes emerged.

In this sample, a majority of physicians seem to recognize the importance of psychosocial factors and the potential value of incorporating mind-body methods in the treatment of a number of common medical conditions. Conditions where there seems to be greater perceived "value-added" from ap- 
proaches such as psychological counseling and other "mind-body" therapies (eg, relaxation, meditation, imagery) include insomnia, headache, and low back pain.

Despite this widespread recognition that psychosocial factors can often play a critical role in understanding the causes of as well as treating, certain health problems, it is important to point out that there are a significant number of physicians who seem to be skeptical of the benefit to be gained from integrating psychosocial factors into medical diagnoses and treatment. Approximately 1 in 3 physicians, for example, indicates that the use of mind-body methods would result in either small or no improvement in clinical outcomes.

It is difficult to determine the extent to which the attitude and practice patterns we identified are generalizable to the larger population of practicing physicians in the United States. Although only speculative, it is possible that our lower than anticipated response rate $(27 \%)$ may have reflected some general lack of interest in or enthusiasm for the topic among the sample of physicians we initially contacted. Such an interpretation seems more likely given our finding that respondents receiving no monetary incentive held somewhat more favorable attitudes toward the role of psychosocial factors than those who received an incentive. In other words, physicians receiving no incentive may have been biased in favor of the topic and hence willing to take part in the survey despite not being compensated. Although we cannot be certain, given this response rate and pattern of responding, it is reasonable to think that physicians in the general population may be less likely to recognize the importance of psychosocial factors than the group of physicians who responded to our survey. One must also consider the possibility that members of the AMA (where we drew our physician sample from) may not be a representative group with respect to attitudes toward mind-body medicine.

Despite the possibility that (for the reasons noted above), our study findings may be painting a somewhat more favorable picture regarding physician attitudes toward the role of mind-body factors than is actually the case, there was still considerable skepticism among respondents in our sample, and this skepticism seems, at least in a number of instances, to be in conflict with the actual evidence base. For example, a recent meta-analysis concluded that the adjunctive use of psychological in- terventions improves clinical outcomes such as pain, function, and quality of life in patients with rheumatoid arthritis. ${ }^{16}$ Similar data exist for osteoarthritis indicating that psychosocial interventions improve treatment outcomes. ${ }^{17,18}$ However, in our sample, only $20 \%$ of physicians (and $12 \%$ of rheumatologists) indicated that the inclusion of psychosocial methods would lead to significant improvements in patients with arthritis.

Similarly, although evidence from randomized controlled trials ${ }^{19-22}$ points to the potential value of mind-body interventions in the management of hypertension and cardiovascular disease, only a minority (36\%) of physicians feel that there would be large value-added if such methods were used as adjunctive treatment for these conditions, although almost one third believe there would be little if any value-added by using such approaches. Among the cardiologists in our sample $(\mathrm{N}=52)$, only 1 in 5 indicate that there would be large value-added if mind-body methods were included as part of the treatment for cardiovascular disease or hypertension.

With respect to future intentions regarding the integration of mind-body approaches, there also seems to be considerable variability. Less than a quarter of physicians express high interest in obtaining further training in such methods, whereas approximately one third indicate that they have little if any interest in receiving additional mindbody training. Again, this variability is reflected in the fact that approximately one third of respondents are either not very or not at all committed to using mind-body approaches in their clinical practice, whereas approximately 1 in 5 are "very committed."

Our multivariate analyses identified several factors that are associated with physicians' attitudes toward mind-body medicine. First, women were considerably more likely than men to be open to the role of psychosocial factors. Second, physicians who report using mind-body methods (eg, relaxation, meditation, imagery) to manage their own health are also more likely to feel that such methods can be valuable as medical treatments.

Physicians' perceptions of the quality of their formal medical training in mind-body methods and the role of psychosocial factors also emerged as a significant predictor of both attitude and practice. Those who report that the training and mentoring they received was not very useful in these areas are 
significantly less open to the value of incorporating mind-body approaches in practice.

In an effort to identify additional barriers, we asked physicians to indicate the extent to which they felt that various factors limited their interest in using mind-body approaches. Multivariate analyses revealed several significant predictors: "insufficient clinic time," "absence of demonstrably effective mind-body therapies," "lack of expertise regarding mind-body methods," and the perception that "psychosocial factors were beyond their capacity to control or influence." These results, which lend empirical support to our previous focus group findings, ${ }^{15}$ suggest that low self-efficacy (to competently address psychosocial issues), perceptions that such factors are frequently difficult to control or impact, lack of knowledge of the evidence base supporting the use of mind-body methods and the role of psychosocial factors in health, and the lack of time to adequately address such issues, are all serving as barriers to medicine's more fully integrating the biopsychosocial perspective.

\section{Policy Implications}

We believe our finding that physicians rate their training in these areas as relatively poor provides strong empirical support for the earlier mentioned Institute of Medicine report, highlighting the need to continue strengthening medical school as well as residency training in the areas of mind-body relationships and the potential impact of psychosocial factors on health. Such a conclusion is further supported by our finding that the majority of physicians report that lack of expertise regarding the use of mind-body methods is a significant limiting factor in their use of such approaches, and that poor training in these areas contributes greatly to the infrequent use of mind-body approaches among physicians.

To increase medicine's integration of the biopsychosocial perspective and to promote appropriate use of/referral to mind-body therapies (eg, relaxation, stress reduction) in clinical practice, our findings suggest 2 other potentially useful strategies. First, it will be important to expose more physicians and physicians-in-training ${ }^{23}$ to the complex interplay of biological, psychological and social factors and their influence on human physiology and health. ${ }^{24-26}$ This need for greater exposure to the evidence base is reflected in the fact that the view held by many physicians' that mind-body methods are not efficacious is actually contradicted by the evidence-base. ${ }^{11,18}$ It is reasonable to conclude that this misperception occurs, at least in part, because physicians are simply unaware of the existing basic science, epidemiologic, and clinical evidence linking mental-emotional factors (eg, stress) to physiologic function and health. This would seem to be an area of continuing medical education that is ripe for further development. ${ }^{27}$

Second, a significant predictor of both attitude toward the role of psychosocial factors in health, and the likelihood that physicians would actually adopt mind-body methods in clinical practice, was the use of such methods to care for their own health. Therefore, as part of educating physicians about the potential clinical value of mind-body methods, it may be important to make the training in these areas experiential as well as didactic, providing physicians with opportunities to actually experiment with (experience the value of) such methods in the "laboratory" of their own lives as a precursor to their introducing patients to them. This idea is supported by studies suggesting that interventions designed to change physician behavior seem most effective when they are not merely didactic in nature but include both active participation and direct experience. ${ }^{14,28}$

Finally, 2 other findings of potential policy significance are physicians' time and issues of reimbursement. The majority of physicians in our survey (more than $70 \%$ ) reported that lack of time (to address psychosocial issues) greatly limited their ability to integrate such factors into their care of patients. This is consistent with other data suggesting that physicians frequently experience managed care practices as negatively impacting the doctorpatient relationship. ${ }^{29}$ In our study, the vast majority (more than $85 \%$ ) also indicated that inadequate reimbursement from health insurers represented another significant obstacle to the utilization of mind-body methods.

From a policy standpoint, these findings suggest that our current health care delivery system may, in many respects, be antithetical to the biopsychosocial model. Specifically, the increasingly restricted time physicians are able to spend with patients may be resulting in a medicine that is, at best, suboptimal, precisely because it limits the extent to which physicians are actually able to adequately address the psychosocial domain of patients lives, either 
diagnostically, or in terms of the actual treatment strategies they employ.

\section{References}

1. Engel GL. The need for a new medical model: A challenge for biomedicine. Science 1977;196:12936.

2. Astin JA. Complementary and alternative medicine and the need for evidence-based criticism. Acad Med Sep 2002;77:864-8; discussion 869-75.

3. Levinson W, Gorawara-Bhat R, Lamb J. A study of patient clues and physician responses in primary care and surgical settings. JAMA 2000;284:1021-7.

4. Marvel MK. Involvement with the psychosocial concerns of patients. Observations of practicing family physicians on a university faculty. Arch Fam Med 1993;2:629-33.

5. Roter DL, Stewart M, Putnam SM, et al. Communication patterns of primary care physicians. JAMA 1997;277:350-6.

6. Levinson W, Roter D. Physicians' psychosocial beliefs correlate with their patient communication skills. J Gen Intern Med 1995;10:375-9.

7. Hall JA, Stein TS, Roter DL, Rieser N. Inaccuracies in physicians' perceptions of their patients. Med Care 1999;37:1164-8.

8. Tresolini CP, Shugars DA. An integrated health care model in medical education: interviews with faculty and administrators. Acad Med 1994;69:231-236.

9. Waldstein SR, Neumann SA, Drossman DA, Novack DH. Teaching psychosomatic (biopsychosocial) medicine in United States medical schools: survey findings. Psychosom Med 2001;63:335-43.

10. Jacobs GD, Pace-Schott EF, Stickgold R, Otto MW. Cognitive behavior therapy and pharmacotherapy for insomnia: a randomized controlled trial and direct comparison. Arch Intern Med Sep 27 2004;164: 1888-96.

11. Astin JA, Shapiro SL, Eisenberg DM, Forys KL. Mind-body medicine: state of the science, implications for practice. J Am Board Fam Pract 2003;16: 131-47.

12. Wolsko PM, Eisenberg DM, Davis RB, Phillips RS. Use of mind-body medical therapies. J Gen Intern Med 2004;19:43-50.

13. Cabana MD, Rand CS, Powe NR, et al. Why don't physicians follow clinical practice guidelines? A framework for improvement. JAMA 1999;282:1458 65.

14. Bero LA, Grilli R, Grimshaw JM, et al. Closing the gap between research and practice: an overview of systematic reviews of interventions to promote the implementation of research findings. The Cochrane Effective Practice and Organization of Care Review Group. BMJ 1998;317:465-8.

15. Astin JA, Goddard T, Forys K. Barriers to the integration of mind-body medicine: Perceptions of phy- sicians, residents and medical students. Explore: J Sci Healing 2005;1:278-83.

16. Astin JA, Beckner W, Soeken K, Hochberg MC, Berman B. Psychological interventions for rheumatoid arthritis: a meta-analysis of randomized controlled trials. Arthritis Rheum Jun 15 2002;47:291302.

17. Lorig K, Holman H. Arthritis self-management studies: a twelve-year review. Health Educ Q 1993; 20:17-28.

18. Astin JA. Mind-body therapies for the management of pain. Clin J Pain 2004;20:27-32.

19. Linden W, Lenz JW, Con AH. Individualized stress management for primary hypertension: a randomized trial. Arch Intern Med 2001;161:1071-80.

20. Schneider RH, Alexander CN, Staggers F, et al. Long-term effects of stress reduction on mortality in persons $\geq 55$ years of age with systemic hypertension. Am J Cardiol 2005;95:1060-4.

21. Schneider RH, Staggers F, Alexander CN, et al. A randomised controlled trial of stress reduction for hypertension in older African Americans. Hypertension 1995;26:820-7.

22. Blumenthal JA, Sherwood A, Babyak M, et al. Effects of exercise and stress management training on markers of cardiovascular risk in patients with ischemic heart disease: A randomized controlled trial. JAMA 2005;293:1626-34.

23. Benbassat J, Baumal R, Borkan JM, Ber R. Overcoming barriers to teaching the behavioral and social sciences to medical students. Acad Med 2003;78: $372-80$.

24. Kiecolt-Glaser JK, McGuire L, Robles TF, Glaser R. Psychoneuroimmunology: psychological influences on immune function and health. J Consult Clin Psychol 2002;70:537-47.

25. Kiecolt-Glaser JK, McGuire L, Robles TF, Glaser R. Emotions, morbidity, and mortality: new perspectives from psychoneuroimmunology. Annu Rev Psychol 2002;53:83-107.

26. Rozanski A, Blumenthal JA, Kaplan J. Impact of psychological factors on the pathogenesis of cardiovascular disease and implications for therapy. Circulation 1999;99:2192-2217.

27. Suls J, Rothman A. Evolution of the biopsychosocial model: prospects and challenges for health psychology. Health Psychol 2004;23:119-125.

28. Davis D, O'Brien MA, Freemantle N, et al. Impact of formal continuing medical education: do conferences, workshops, rounds, and other traditional continuing education activities change physician behavior or health care outcomes?. JAMA 1999;282: $867-74$.

29. Simon SR, Pan RJD, Sullivan AM, et al. Views of managed care: a survey of students, residents, faculty, and deans at medical schools in the United States. N Engl J Med 1999;340:928-36. 